\title{
A Minimal Polynomial Basis Solution to Residual Generation for Fault Diagnosis in Linear Systems
}

\author{
Erik Frisk, Mattias Nyberg \\ email:frisk@isy.liu.se,matny@isy.liu.se
}

\begin{abstract}
A fundamental part of a fault diagnosis system is the residual generator. Here a new method, the minimal polynomial basis approach, for design of residual generators for linear systems, is presented. The residual generation problem is transformed into a problem of finding polynomial bases for null-spaces of polynomial matrices. This is a standard problem in established linear systems theory, which means that numerically efficient computational tools are generally available. It is shown that the minimal polynomial basis approach can find all possible residual generators, including those of minimal McMillan degree, and the solution has a minimal parameterization. It is shown that some other well known design methods, do not have these properties.
\end{abstract}

Keywords: fault detection, diagnosis, polynomial bases, residual generation, perfect decoupling, disturbance decoupling 


\section{Introduction}

This report is a study of linear residual generation for linear systems with no model uncertainties. A general linear residual generator can be written

$$
r=Q(s)\left(\begin{array}{l}
y \\
u
\end{array}\right)
$$

where $Q(s)$ is a linear, finite-dimensional, proper filter. A number of methods for designing linear residual generators have been proposed in literature, see for example (Patton and Kangethe, 1989; Wünnenberg, 1990; White and Speyer, 1987; Massoumnia, Verghese and Willsky, 1989; Nikoukhah, 1994; Chow and Willsky, 1984; Nyberg and Nielsen, 1997; Liu and Si, 1997). All these methods are methods to design the transfer matrix $Q(s)$. Four natural questions that have not been explicitly raised before are the following:

- Does the method find all possible residual generators?

- Does the method find residual generators of minimal order?

- Does the solution represent a minimal parameterization or is it over parameterized?

- Is the design freedom clearly parameterized?

The four main questions are shown to be naturally handled by formulating the residual generation problem in the standard framework of polynomial matrices. Also, a design focus is maintained in the sense that it is assured that a numerically feasible design algorithm is generally available. The outcome of this is a method, the Minimal Polynomial Basis Approach, which is presented in Section 2. With this approach, the decoupling problem is transformed into finding a minimal basis to a polynomial matrix. The motivation for this transformation to a purely polynomial problem is that there exists well established theory (Kailath, 1980; Lancaster and Tismenetsky, 1985) regarding polynomial matrices. In addition, the generally available Polynomial Toolbox (Henrion, Kraffer, Kwakernaak, M.Sebek and Strijbos, 1997) for MATLAB contains an extensive set of tools for numerical handling of polynomial matrices. Some relations to other design methods are explored in Section 4 where it is shown that issues regarding minimality and completeness is not at all obvious for other methods.

\section{The Minimal Polynomial Basis Approach}

This chapter introduces the minimal polynomial basis approach to the design of linear residual generators. The approach is based on intuitive concepts from linear algebra such as bases and null-spaces for polynomial and rational spaces. It does not adopt, as is common in the fault diagnosis community, an observer view, e.g. like the Unknown Input Observer and Eigenstructure assignment of observers methods of designing residual generators. This is because the primary 
issues of this chapter is to handle completeness of solution and minimality which are more easily addressed in a polynomial basis framework. Also, the freedom available to the design engineer is clear which is important when performing a design.

\subsection{Problem Formulation}

The systems studied are on the form

$$
y=G(s) u+H(s) d+L(s) f
$$

where $y$ is measurements, $u$ is known inputs to the system, $d$ is unknown disturbances including the non-monitored faults, and $f$ is the rest of the faults. The filter $Q(s)$ is restricted to linear, finite-dimensional, and proper filters. The filter $Q(s)$ in (1) form a residual generator if and only if $r(t)=0$ for all $d(t)$ and $u(t)$ when $f(t)=0$, i.e. the control signal and all disturbances are decoupled from the residual. To be able to detect faults, it is also required that $r(t) \neq 0$ when $f(t) \neq 0$. Inserting (2) into (1) gives

$$
r=Q(s)\left[\begin{array}{cc}
G(s) & H(s) \\
I & 0
\end{array}\right]\left[\begin{array}{l}
u \\
d
\end{array}\right]+Q(s)\left[\begin{array}{c}
L(s) \\
0
\end{array}\right] f
$$

To make $r(t)=0$ when $f(t)=0$, i.e. for $Q(s)$ to form a residual generator it must hold that

$$
Q(s)\left[\begin{array}{cc}
G(s) & H(s) \\
I & 0
\end{array}\right]=0
$$

which is equivalent with $Q(s)$ must belong to the left null-space of

$$
M(s)=\left[\begin{array}{cc}
G(s) & H(s) \\
I & 0
\end{array}\right]
$$

This rational null-space is denoted $\mathcal{N}_{l}(M(s))$ and a corresponding basis for the null-space is denoted $N_{M}(s)$ where the rows are the base vectors. This basis is not unique, in fact it might consist of polynomial base vectors, i.e. rational functions without a denominator. Such strictly polynomial bases will be of particular interest during the remaining parts of this chapter. Definitions of order and other properties of bases, minimal polynomial bases are included in Appendix A.

In the design of residual generators, the two main objectives are then:

1. Find $Q(s)$ such that $Q(s) M(s)=0$

2. Find $Q(s)$ such that $Q(s)\left[\begin{array}{c}L(s) \\ 0\end{array}\right]$ has desired properties to ensure good fault detectability properties. 
where the residual generator $Q(s)$ is of least order. These two problems can be solved by first finding all $Q(s) \in \mathcal{N}_{l}(M(s))$ and then choosing within this set of residual generators those who have good fault detectability properties. Note that breaking up the problem in two independent steps is only permitted if all $Q(s)$ is found in the first step. Thus, in the first step of the design of the residual generator $Q(s)$ we never need to consider $f$ or $L(s)$. The first step can be achieved by finding a basis for the rational vector-space $\mathcal{N}_{l}(M(s))$.

\subsection{Polynomial Bases for Rational Vector-spaces}

A basis for a rational vector space follows notions from basic linear algebra. For rational vector spaces, such as $\mathcal{N}_{l}(M(s))$, it is perhaps natural to consider rational matrices to represent its basis. However, among all bases for a given rational vector space, certain bases are entirely polynomial which is showed in Theorem 1.

Theorem 1. A polynomial basis exists for any rational vector-space $\mathcal{F}$.

Proof. Let the rows of the rational matrix $F(s)$ be a rational basis for $\mathcal{F}$. Then, for any $y(s) \in \mathcal{F}$ there exists a rational $x(s)$ such that

$$
y(s)=x(s) F(s)
$$

Perform a left MFD over polynomial matrices on $F(s)$, i.e. find polynomial matrices $N(s)$ and $D(s)$ such that $F(s)=D^{-1}(s) N(s)$ and $D(s)$ is a full-rank square matrix. Then it holds that

$$
y(s)=x(s) D^{-1}(s) D(s) F(s)=x(s) D^{-1}(s) N(s)=\tilde{x}(s) N(s)
$$

i.e. the polynomial matrix $N(s)$ is a basis for the rational vector-space $\mathcal{F}$.

However, some caution is needed when an expression for all polynomial vectors in a rational vector-space is needed.

\section{Example 2.1}

Let the rows of the matrix $F(s)$ be a basis for the rational vector-space $\mathcal{F}$.

$$
F(s)=\left[\begin{array}{ccc}
s & 1 & 0 \\
0 & 1 & -s \\
1 & 0 & 2
\end{array}\right]
$$

It is clear that it is a basis since $\operatorname{det}(F(s)) \neq 0$, i.e. the matrix has full rank and therefore are the rows linearly independent. It is also clear that the vector

$$
u(s)=\left[\begin{array}{lll}
1 & 0 & 0
\end{array}\right] \in \mathcal{F}
$$

However, there exists no polynomial $x(s)$ such that $u(s)=x(s) F(s)$, rational $x(s)$ is needed. 
However, if the basis is irreducible, i.e. full rank for all $s$, then according to Theorem 10 all polynomial vectors has polynomial co-efficients $x(s)$. This is important to note when a basis for all polynomial vectors in a space is used. Other properties of vector spaces used in this thesis is found in Appendix A.

The use of bases ensures a simple parameterization of all residual generators, which was one of the four main issues presented in the beginning of this chapter. Also, finding residual generators of the least order (number of states) was stated as an important issue. This makes minimal bases of particularly interesting. In (Forney, 1975; Kailath, 1980) such minimal bases are defined.

Definition 1 (Minimal Polynomial Basis). Let $\mathcal{F}$ be a $k$-dimensional rational vector space. A minimal basis for $\mathcal{F}$ is a $k \times n$ matrix $V(s)$ such that the rows of $V(s)$ is a basis for $\mathcal{F}$ and has the least order of all polynomial bases for $\mathcal{F}$.

The next section will assume that the general problem of finding $N_{M}(s)$ is solved and focus on how this basis can be used to form a residual generator for fault diagnosis. The problem of finding $N_{M}(s)$ is then revisited in Section 3 where a computationally simple, efficient, and numerically stable method from established theory on linear systems, is presented.

\subsection{Forming the Residual Generator}

From now on, the basis $N_{M}(s)$ will always be a minimal polynomial basis unless otherwise stated. When a minimal polynomial basis $N_{M}(s)$ have been obtained, the second and final step in the residual generator design is to shape fault-toresidual responses as described next.

The minimal polynomial basis $N_{M}(s)$ is, by Theorem 9, irreducible and then, according to Theorem 10, all decoupling polynomial matrices $F(s)$, i.e. all polynomial matrices $F(s)$ such that $F(s) M(s)=0$, can be parameterized as

$$
F(s)=\phi(s) N_{M}(s)
$$

where $\phi(s)$ is a polynomial matrix of suitable dimensions. This parameterization matrix $\phi(s)$ can e.g. be used to shape the fault-to-residual response or simply to select one row in $N_{M}(s)$.

When a decoupling polynomial matrix has been selected for implementation to form a residual generator it must be made realizable since a polynomial matrix represents an improper transfer matrix and thus not realizable. If the decoupling polynomial matrix is $F(s)$, then a realizable rational transfer matrix $Q(s)$, i.e. the residual generator, can be formed as

$$
Q(s)=D_{F}^{-1}(s) F(s)
$$

where the rows in the invertible polynomial matrix $D_{F}(s)$ has greater or equal degree than the corresponding row-degree in $F(s)$ (Theorem 6.3-12 in (Kailath, 1980)). The degree constraint is the only constraint on $D_{F}(s)$. This means that the dynamics, i.e. poles, of the residual generator $Q(s)$ can be chosen freely. 
This also means that the minimal order of a realization of a decoupling filter is determined by the row-degrees of $N_{M}(s)$. This clearly shows that the order of polynomial bases, as defined in Definition 7 , is a suitable measure to minimize when searching for residual generators of the least order. This since the rowdegrees of the basis directly gives the minimum order of the resulting residual generator. Now follows a theorem that motivates the parameterization (5) and shows that it is a parameterization that parameterizes all residual generators.

Theorem 2. For a rational matrix $Q(s)$ it holds that $Q(s) M(s)=0$ if and only if it there exists polynomial matrices $\phi(s)$ and $D_{F}(s)$ such that

$$
Q(s)=D_{F}^{-1}(s) \phi(s) N_{M}(s)
$$

where $N_{M}(s)$ is an irreducible basis for the left null-space of $M(s)$.

Proof.

if part:

Assume that polynomial matrices $\phi(s)$ and $D_{F}(s)$ exists such that $Q(s)=D_{F}^{-1}(s) \phi(s) N_{M}(s)$. Then

$$
Q(s) M(s)=D_{F}^{-1}(s) \phi(s) N_{M}(s) M(s)=D_{F}^{-1}(s) \phi(s) 0=0
$$

\section{only-if part:}

Now assume $Q(s) M(s)=0$. Any rational $Q(s)$ can always, via an MFD over polynomial matrices, be rewritten as

$$
Q(s)=D_{F}^{-1}(s) N_{Q}(s)
$$

where $D_{F}(s)$ and $N_{Q}(s)$ are polynomial matrices. Then it holds that

$$
D_{F}(s) Q(s) M(s)=N_{Q}(s) M(s)=0
$$

i.e. $N_{Q}(s)$ is a polynomial matrix in $\mathcal{N}_{l}(M(s))$. According to Theorem 10 , there exists a polynomial matrix $\phi(s)$ such that $N_{Q}(s)=\phi(s) N_{M}(s)$. Now it is clear that

$$
Q(s)=D_{F}^{-1}(s) \phi(s) N_{M}(s)
$$

which ends the proof.

Therefore, any residual generator $Q(s)$ can be expressed with the polynomial matrices $\phi(s)$ and $D_{F}(s)$. To limit the solution to e.g. a stable and proper solution for implementation, impose the degree constraint on $D_{F}(s)$ and select poles in the left half-plane.

\subsection{Approach Summary}

The main advantage in viewing the disturbance decoupling problem as finding a polynomial basis for the left null-space of $M(s)$ is that issues regarding minimality, and whether the method finds all decoupling residual generators are 
naturally handled. All derivations above is performed in the continuous case. However, the continuous case can be transferred to the time-discrete case by substituting $z$ for $s$ and improper for non-causal.

The Minimal Polynomial Basis approach can be summarized by the following steps:

1. Find a minimal polynomial basis for $\mathcal{N}_{l}(M(s))$ denoted $N_{M}(s)$.

2. Select parameterization matrix $\phi(s)$ and $D_{F}(s)$ to shape residual-to-fault response and obtain a proper residual generator $Q(s)$ as

$$
Q(s)=D_{F}^{-1}(s) \phi(s) N_{M}(s)
$$

Fault response is evaluated by studying the transfer matrix

$$
G_{r f}(s)=Q(s)\left[\begin{array}{c}
L(s) \\
0
\end{array}\right]
$$

Note that all freedom available is condensed into the two matrices $D_{F}(s)$ and $\phi(s)$ that can be chosen arbitrarily except for the degree constraint on $D_{F}(s)$ and stability restrictions if an implementable $Q(s)$ is sought. It is clear that the matrix formed by $D_{F}^{-1}(s) \phi(s)$ can be selected, e.g. as a LP-filter, to filter out measurement noise. If the designer has any prior knowledge on frequency distribution of the faults, $D_{F}(s)$ and $\phi(s)$ can be used to emphasize these frequency ranges.

\section{Methods to find Minimal Polynomial Basis to $\mathcal{N}_{l}(M(s))$}

The problem of finding a minimal polynomial basis to the left null-space of the rational matrix $M(s)$ can be solved by transforming the rational problem to a problem of finding a minimal polynomial basis to the left null-space of a polynomial matrix. This transformation can be done in several different ways. In this section two possibilities are demonstrated, one that is useful when the system models are available in input-output form (transfer matrices) and one that is useful when the model is available in state-space form. Also included is a description on how to compute a basis for the null-space of a polynomial matrix.

\subsection{Frequency Domain Solution}

If the system model is given as a transfer matrix, a way of transforming the rational problem to a polynomial problem is to perform a right MFD over polynomial matrices on $M(s)$, i.e.

$$
M(s)=\widetilde{M}_{1}(s) \widetilde{D}^{-1}(s)
$$


One simple example is

$$
M(s)=\widetilde{M}_{1}(s) d^{-1}(s)
$$

where $d(s)$ is the least common multiple of all denominators. By finding a polynomial basis for the left null-space of the polynomial matrix $\widetilde{M}_{1}(s)$, a basis is also found for the left null-space of $M(s)$. Since $\widetilde{D}(s)$ (e.g. $d(s)$ ) is square and of full normal rank, i.e. the left null-space of $\widetilde{D}(s)$ is empty, it is clear that no solutions are missed during the transformation. Thus the problem of finding a minimal polynomial basis to $\mathcal{N}_{l}(M(s)$ ) has been transformed into finding a minimal polynomial basis to $\mathcal{N}_{l}\left(\widetilde{M}_{1}(s)\right)$.

\section{$3.2 \quad$ State-Space Solution}

If the system model is available in state-space form, it is desired to address the problem directly with the state-space matrices instead of transforming the model into an MFD as above. It is here showed that the system matrix in statespace form (Rosenbrock, 1970) can be used to find the left null-space of $M(s)$. The system matrix has been used before in the context of fault diagnosis, see e.g. (Nikoukhah, 1994; Magni and Mouyon, 1994).

Assume that the system is described in state-space form,

$$
\begin{aligned}
\dot{x}(t) & =A x(t)+B_{u} u(t)+B_{d} d(t) \\
y(t) & =C x(t)+D_{u} u(t)+D_{d} d(t)
\end{aligned}
$$

where $x$ is the $n$-dimensional state. Denote the system matrix $M_{s}(s)$,

$$
M_{s}(s)=\left[\begin{array}{cc}
C & D_{d} \\
-(s I-A) & B_{d}
\end{array}\right]
$$

Define the matrix $P$ as

$$
P=\left[\begin{array}{ll}
I & -D_{u} \\
0 & -B_{u}
\end{array}\right]
$$

Then the following theorem gives a direct method on how to find a minimal polynomial basis to $\mathcal{N}_{l}(M(s))$ via the system matrix.

Theorem 3. Let $V(s)$ be minimal polynomial basis for $\mathcal{N}_{l}\left(M_{s}(s)\right)$ and let the pair $\left\{A,\left[B_{u} B_{d}\right]\right\}$ be controllable. Then $W(s)$ obtained as $W(s)=V(s) P$, is a minimal polynomial basis for $\mathcal{N}_{l}(M(s))$.

Before this theorem can be proved, a lemma is needed. Define transformations

$$
\begin{aligned}
W(s) & =V(s) P \\
V(s) & =W_{1}(s)\left[I C(s I-A)^{-1}\right]
\end{aligned}
$$

where $W(s)=\left[W_{1}(s) W_{2}(s)\right]$ and $W_{1}(s)$ is the first $n$ (number of states) columns in $W(s)$. 
Lemma 4. Let the matrices $W(s)$ and $V(s)$ be related by the transformations (8) and (9), and assume that the pair $\left\{A,\left[B_{u} B_{d}\right]\right\}$ is controllable. Then, $W(s)$ is polynomial, irreducible, and $W(s) \in \mathcal{N}_{l}(M(s))$ if and only if $V(s)$ is polynomial, irreducible, and $V(s) \in \mathcal{N}_{l}\left(M_{s}(s)\right)$.

\section{Proof.}

if-part

In the if-part of the proof it is assumed that $V(s)$ is polynomial, irreducible, and $V(s) \in \mathcal{N}_{l}\left(M_{s}(s)\right)$.

First consider the following relation between the matrices $M(s)$ and $M_{s}(s)$ :

$$
\begin{aligned}
P M(s)\left(\begin{array}{l}
u \\
d
\end{array}\right) & =\left[\begin{array}{ccc}
C(s I-A)^{-1} B_{u} & C(s I-A)^{-1} B_{d}+D_{d} \\
-B_{u} & 0 & 0
\end{array}\right]\left(\begin{array}{l}
u \\
d
\end{array}\right)= \\
& =\left[\begin{array}{cc}
C & D_{d} \\
-(s I-A) & B_{d}
\end{array}\right]\left[\begin{array}{cc}
(s I-A)^{-1} B_{u} & (s I-A)^{-1} B_{d} \\
0 & I
\end{array}\right]\left(\begin{array}{l}
u \\
d
\end{array}\right)= \\
& =M_{s}(s)\left(\begin{array}{l}
x \\
d
\end{array}\right)
\end{aligned}
$$

If $V(s) M_{s}(s)=0$, then since the signals $u(t)$ and $d(t)$ can be chosen arbitrarily, $W(s) M(s)$ must also be 0 , i.e. $W(s) \in \mathcal{N}_{l}(M(s))$. It is also immediate that if $V(s)$ is polynomial, $W(s)=V(s) P$ is also polynomial.

Left to prove is that $W(s)$ is irreducible which is done by contradiction. It is clear that the following relation must hold:

$$
V(s)\left[P M_{s}(s)\right]=V(s)\left[\begin{array}{cccc}
I & -D_{u} & C & D_{d} \\
0 & -B_{u} & -(s I-A) & B_{d}
\end{array}\right]=[W(s) 0]
$$

Considering the matrix $\left[P M_{s}(s)\right]$, if the system is controllable, then the PBH rank test in Theorem 7 implies that the lower part of this matrix has full rank for all $s$. The irreducibility of the lower row together with the shape of the first block-column of $\left[P M_{s}(s)\right]$ gives that the whole matrix is irreducible. Now assume that $W(s)$ is not irreducible, i.e. there is a $s_{0}$ such that $W\left(s_{0}\right)$ does not have full row-rank. This means that there exists a row-vector $\gamma \neq 0$ such that $\gamma V\left(s_{0}\right)\left[P M_{s}\left(s_{0}\right)\right]=\gamma\left[W\left(s_{0}\right) 0\right]=0$. Since $\left[P M_{s}\left(s_{0}\right)\right]$ has full row-rank it must hold that $\gamma V\left(s_{0}\right)=0$ and $V(s)$ cannot be irreducible. This contradiction implies that $W(s)$ must be irreducible, which concludes the if-part of the proof.

\section{only-if-part}

In the only-if part of the proof it is assumed that $W(s)$ is polynomial, irreducible, and $W(s) \in \mathcal{N}_{l}(M(s))$.

From transformation (9)

$$
\begin{aligned}
V(s) M_{s}(s) & =W_{1}(s)\left[\begin{array}{ll}
I & C(s I-A)^{-1}
\end{array}\right]\left[\begin{array}{cc}
C & -D_{d} \\
-(s I-A) & -B_{d}
\end{array}\right]= \\
& =\left[\begin{array}{ll}
0 & W_{1}(s)\left(-D_{d}-C(s I-A)^{-1} B_{d}\right)
\end{array}\right]=0
\end{aligned}
$$


The last equality is because $W_{1}(s) H(s)=0$. This shows that $V(s) \in \mathcal{N}_{l}\left(M_{s}(s)\right)$.

Now, from the discussion in the if-part of the proof, it is clear that (10) holds with $\left[P M_{s}(s)\right]$ irreducible. Then from Theorem 8 it is clear that $V(s)$ is polynomial.

To show that $V(s)$ is irreducible, assume that $V(s)$ is not irreducible, i.e. there exists a $s_{0}$ such that $V\left(s_{0}\right)$ does not have full row-rank. This means that there exists a row-vector $\gamma \neq 0$ such that $\gamma V\left(s_{0}\right)=0$. Once again using (10) gives

$$
\gamma\left[W\left(s_{0}\right) 0\right]=\gamma V\left(s_{0}\right)\left[P M_{s}\left(s_{0}\right)\right]=0
$$

i.e. and $\gamma W\left(s_{0}\right)=0$ which is a contradiction to the assumption that $W(s)$ was irreducible. This contradiction implies that $V(s)$ must be irreducible, which concludes the only if part and also the proof.

Now, return to the proof of Theorem 3.

Proof. Assume that the dimension of $\mathcal{N}_{l}\left(M_{s}(s)\right)$ is $p$. Then $V(s)$ will have $p$ rows. A $W(s)$ obtained from (8) will also have $p$ rows. Further Lemma 4 implies that $W(s)$ have full row rank and $W(s) M(s)=0$, i.e. $W(s)$ is a basis for a $p$-dimensional space in the left null-space of $M(s)$. Left to show is that it is a basis for the whole null-space, i.e. that the dimension of $\mathcal{N}_{l}(M(s))$ also is $p$. Now assume that $W(s)$ is not a basis for $\mathcal{N}_{l}(M(s))$. This would mean that $\operatorname{dim} \mathcal{N}_{l}(M(s))>p$. In that case, let $W^{\prime}(s)$ be a basis for $\mathcal{N}_{l}(M(s))$ with $p^{\prime}>p$ rows, and obtain $V^{\prime}(s)$ in accordance with (9). Lemma 4 implies that $V^{\prime}(s)$ would have full row rank and also that $V^{\prime}(s) M_{s}(s)=0$. Since $V^{\prime}(s)$ has $p^{\prime}>p$ rows, this would imply that $\operatorname{dim} \mathcal{N}_{l}\left(M_{s}(s)\right)>p$, which is a contradiction. Therefore $W(s)$ must be an irreducible (from Lemma 4) basis for $\mathcal{N}_{l}(M(s)$ ).

The matrix $W(s)$ is now proven to be a polynomial, irreducible basis for $\mathcal{N}_{l}(M(s))$. According to Theorem 9, the only thing left to prove is that the basis $W(s)$ is row-reduced. Partition $V(s)=\left[V_{1}(s) V_{2}(s)\right]$ according to the partition of $M_{s}(s)$. Let

$$
\begin{aligned}
& V_{1}(s)=S_{1}(s) V_{1, h r}+q_{1}(s) \\
& V_{2}(s)=S_{2}(s) V_{2, h r}+q_{2}(s)
\end{aligned}
$$

The matrices $S_{i}(s)$ is diagonal matrices with diagonal elements $s^{k_{i j}}$ where $k_{i j}$ is the row-degrees of $V_{i}(s)$. The constant matrices $V_{i, h r}$ is the high-order coefficient matrix and $q_{i}(s)$ is the rest polynomial. Since $V(s) \in \mathcal{N}_{l}\left(M_{s}(s)\right)$, it holds that $V_{1}(s) C=V_{2}(s)(s I-a)$, i.e.

$$
\begin{aligned}
S_{1}(s) V_{1, h r} C+q_{1}(s) C & =S_{2}(s) V_{2, h r}(s I-A)+q_{2}(s)(s I-A) \\
& =s S_{2}(s) V_{2, h r}+\tilde{q}_{2}(s)
\end{aligned}
$$

By identifying the highest order terms on each side it is immediate that $s S_{2}(s)=$ $S_{1}(s)$, i.e. each row in $V_{2}(s)$ has lower degree than the corresponding row in $V_{1}(s) C$. It also holds that the row degrees in $V_{1}(s) C$ has less or equal row 
degrees than $V_{1}(s)$ since $C$ is a constant matrix. Thus, the row degrees in $V_{2}(s)$ has less degree than the corresponding row in $V_{1}(s)$. Since $V(s)$ is a minimal polynomial basis, it is row reduced. That is the high-order coefficient matrix for $V(s)$ has full row rank. From this follows that $V_{1, h r}$ has full row rank.

From the definition of $P$ it follows that

$$
\left[W_{1}(s) W_{2}(s)\right]=\left[V_{1}(s) \quad\left(-V_{1}(s) D_{u}-V_{2}(s) B_{u}\right)\right]
$$

From the degree discussion above it follows that the high-order coefficient matrix of $W(s)$ looks like $W_{h r}=\left[V_{1, h r} \star\right]$, which obviously has full row-rank, i.e. $W(s)$ is row reduced. Thus we have shown that $W(s)$ is an irreducible basis and column reduced, which implies that it is a minimal polynomial basis.

In conclusion, as in the previous section, the problem of finding a minimal polynomial basis to $\mathcal{N}_{l}(M(s)$ ) has been transformed into finding an minimal polynomial basis to a polynomial matrix, in this case the system matrix $M_{s}(s)$.

\subsection{Finding the minimal polynomial basis}

Now the final problem is to find a minimal polynomial basis to a polynomial matrix. It is noteworthy that, by inspection of (3), it holds that the dimension (number of rows) of $N_{M}(s)$ is less or equal to $m$ (number of measurements) with equality when there are no disturbances. It is also immediate that the number of independent disturbances that can be decoupled is limited to $m-1$.

In the general case, no explicit expressions for the basis and/or the order of the basis is available. However, the next section will discuss the case with no disturbances where general statements are easily available. After this special case, standard algorithms to extract the sought basis in the general case is presented.

\section{No Disturbance Case}

If there are no disturbances, i.e. $H(s)=0$, the matrix $M(s)$ gets a simpler structure

$$
M_{n d}(s)=\left[\begin{array}{c}
G(s) \\
I
\end{array}\right]
$$

A minimal polynomial basis for the left null-space of $M_{n d}(s)$ is particularly simple due to the special structure and a minimal basis is then given directly by the following theorem.

Theorem 5. If $G(s)$ is a proper transfer matrix and $\bar{D}_{G}(s), \bar{N}_{G}(s)$ form an irreducible left MFD over polynomial matrices, i.e. $\bar{N}_{G}(s)$ and $\bar{D}_{G}(s)$ are left co-prime polynomial matrices and $G(s)=\bar{D}_{G}^{-1}(s) \bar{N}_{G}(s)$. Then,

$$
N_{M}(s)=\left[\bar{D}_{G}(s)-\bar{N}_{G}(s)\right]
$$


form a minimal basis for the left null-space of the matrix

$$
M(s)=\left[\begin{array}{c}
G(s) \\
I
\end{array}\right]
$$

Proof. The proof can be found in (Kailath, 1980).

Here, the dimension of the null-space is $m$, i.e. the number of measurements, and the order of the minimal base is given by the following theorem.

Theorem 6. The set of observability indices of a transfer function $G(s)$ is equal to the set of row degrees of $\bar{D}_{G}(s)$ in any row-reduced irreducible left MFD $G(s)=\bar{D}_{G}^{-1}(s) \bar{N}_{G}(s)$.

Proof. A proof of the dual problem, controllability indices, can be found in (Chen, 1984) (p. 284).

Thus, a minimal polynomial basis for matrix $M_{n d}(s)$ is given by a left MFD of $G(s)$ and the order of the basis is the sum of the observability indices of $G(s)$. Remark: The result (12) implies that finding the left null-space of the rational transfer matrix (3), in the general case with disturbances included, can be reduced to finding the left null-space of the rational matrix

$$
\widetilde{M}_{2}(s)=\bar{D}_{G}(s) H(s)
$$

Therefore, this is an alternative to the use of the matrix $\widetilde{M}_{1}(s)$ in $(6)$. This view closely connects with the so called frequency domain methods, which are further examined in Section 4.

\section{Finding a Minimal Polynomial Basis to a General Polyno- mial Matrix}

For the general case, including disturbances, the only remaining problem is how to find a minimal polynomial basis to a general polynomial matrix. This is a well-known problem in literature on linear systems and a number of different algorithms exist. In this chapter, two algorithms will be presented. The first is based on the Hermite form (Kailath, 1980) and a second algorithm is based on the polynomial echelon form (Kailath, 1980). Both methods are implemented in the Polynomial Toolbox (Henrion et al., 1997) for MatLaB.

The two algorithms have very different numerical properties. Although the algorithm based on Hermite form is easy to understand, simulations have shown it to be numerically unstable. It is included to gain some basic understanding of the problem. However the algorithm based on polynomial echelon form is both fast and numerically stable and should be the preferred choice. 


\section{The Hermite Form Algorithm}

Any polynomial matrix can be transformed into column Hermite form by elementary row operations. Assume $M(s)$ is a $p \times q$ matrix. Then there exists a $p \times p$, unimodular matrix $U(s)=\left[U_{1}^{T}(s) U_{2}^{T}(s)\right]^{T}$ such that

$$
\left[\begin{array}{l}
U_{1}(s) \\
U_{2}(s)
\end{array}\right] M(s)=\left[\begin{array}{c}
R(s) \\
0
\end{array}\right]
$$

where $R(s)$ is a $(p-r) \times q$ matrix and $r$ is the normal rank of $M(s)$. The, non-unique, matrix $U(s)$ can be found e.g. as described in Theorem 6.3-2 in (Kailath, 1980). The last $r$ rows in $U(s)$, i.e. $U_{2}(s)$, thus spans the left null-space of $M(s)$. The matrix $U_{2}(s)$ is irreducible because $U(s)$ is unimodular. $U_{2}(s)$ is however not necessary row-reduced, i.e. $U_{2}(s)$ is not necessarily a minimal basis. However, $U_{2}(s)$ can be made row-reduced by elementary row operations. This is best illustrated with an example that shows the main idea and also illustrates how the minimality property is connected with the row-reduced property.

\section{Example 3.2}

Consider the polynomial matrix $M(s)$ with rank $r=2$

$$
M(s)=\left[\begin{array}{ccc}
1 & 0 & -s \\
0 & s^{3}+2 s^{2}+s & s^{3}+2 s^{2}+s \\
s & s^{3}+2 s^{2}+s & s^{3}+s^{2}+s \\
s^{2} & 0 & -s^{3}
\end{array}\right]
$$

The column Hermite form of $M(s)$ is

$$
\left[\begin{array}{cccc}
1 & 0 & 0 & 0 \\
0 & 1 & 0 & 0 \\
-s & -1 & 1 & 0 \\
-s^{2} & 0 & 0 & 1
\end{array}\right] M(s)=\left[\begin{array}{ccc}
1 & 0 & -s \\
0 & s+2 s^{2}+s^{3} & s+2 s^{2}+s^{3} \\
0 & 0 & 0 \\
0 & 0 & 0
\end{array}\right]
$$

Here, the last two rows of $U(s)$ form a basis for the left null-space of $H(s)$ and is denoted $F(s)$.

$$
F(s)=\left[\begin{array}{cccc}
-s & -1 & 1 & 0 \\
-s^{2} & 0 & 0 & 1
\end{array}\right]
$$

The matrix $F(s)$ is obviously irreducible, it is however not row-reduced because the high-coefficient matrix $F_{h r}$

$$
F_{h r}=\left[\begin{array}{llll}
-1 & 0 & 0 & 0 \\
-1 & 0 & 0 & 0
\end{array}\right]
$$

is not of full rank. However, by multiplication from the left with a suitably chosen unimodular matrix, $F(s)$ can be made row-reduced. General algorithms to find the unimodular matrix making $F(s)$ row-reduced is available, e.g. (Callier, 1985). In the example above,

$$
\left[\begin{array}{ll}
-1 & 0 \\
-s & 1
\end{array}\right] F(s)=\left[\begin{array}{llll}
s & 1 & -1 & 0 \\
0 & s & -s & 1
\end{array}\right]=F_{\min }(s)
$$


The matrix $F_{\min }(s)$ is both irreducible and row-reduced, and accordingly to Theorem 9 , it is a minimal basis for the left null-space.

\section{The Polynomial Echelon Form Algorithm}

The polynomial echelon form method is described in (Kailath, 1980; Kung, Kailath and Morf, 1977). Now follows a very brief description of the algorithm to illustrate the algorithm usage and computational complexity.

Consider the polynomial equation

$$
F(s) M(s)=0
$$

Assume that the polynomial basis $F(s)$ is in the canonical polynomial echelon form. This assumption is not restrictive since all full row rank polynomial matrices can be transformed to polynomial echelon form by elementary row operations, i.e. by multiplication from the left with a unimodular matrix. The left hand side of (14) can be rewritten as

$$
F(s) M(s)=\left(F_{0}+F_{1} s+\ldots F_{\nu} s^{\nu}\right) M(s)=\left[F_{0} \ldots F_{\nu}\right]\left[\begin{array}{c}
M(s) \\
s M(s) \\
\vdots \\
s^{\nu} M(s)
\end{array}\right]=\widetilde{F} \mathcal{M}(s)
$$

which also defines $\mathcal{M}(s)$ and the coefficient matrix $\widetilde{F}$. The order $\nu$ is not known a priori but is limited to $\nu \leq(p-1) \operatorname{deg} M(s)$ (Henrion et al., 1997), where $\operatorname{deg} M(s)$ denotes the maximum row (and column) degree of $M(s)$. By examining the rows of $\mathcal{M}(s)$, from top to bottom, the rows can be classified as independent rows or dependent rows. Independent and dependent rows can equally well be determined from the coefficient matrix $\widetilde{\mathcal{M}}$. (The matrix $\widetilde{\mathcal{M}}$ is known as the Generalized Resultant matrix of $M(s)$.)

Since $F(s)$ is in polynomial echelon form, the rows of $\widetilde{F}$ must define a set of primary dependent rows in $\mathcal{M}(s)$. Of all sets of primary dependent rows, the set defined by $\widetilde{F}$ must be of minimal order, i.e. there is no other set of primary dependent rows, containing the same number of rows and with lower degrees.

Each set of primary dependent rows spans a subspace of $\mathcal{N}_{l}(M(s))$. Therefore, since $F(s)$ spans the whole left null-space of $\mathcal{M}(s)$, the set of primary dependent rows defined by $\widetilde{F}$, must be of largest possible size.

With these statements in mind, we know that the matrix $\widetilde{F}$, and also $F(s)$, can be found by searching, from top to bottom, in $\widetilde{\mathcal{M}}$ for the largest uppermost set of primary dependent rows. The search for primary dependent rows in $\widetilde{\mathcal{M}}$ can be performed by using elimination methods or projection methods. Projection methods are a better choice since they are more numerically stable (Kung et al., 1977). See also Section 3.3 for further elaborations on this topic.

It has been assumed that the search for primary dependent rows is performed in such a way that a canonical polynomial echelon form is obtained. In this way, the minimal basis obtained will be unique. For most purposes, this uniqueness 
is not important, which makes it sufficient to perform the row search such that a quasi-canonical polynomial echelon form is obtained.

When performing the search for primary dependent rows, it is important to know when to stop. There are two possibilities. The first is that we know the rank of $M(s)$. Then the largest set of primary dependent rows will contain $p-\operatorname{rank} M(s)$ rows. The other possibility is to use $\nu=(p-1) \operatorname{deg} M(s)$ when constructing the matrix $\mathcal{M}(s)$. Then the set of primary dependent rows of minimal order must be contained in $\mathcal{M}(s)$. Now follows an example to illustrate the calculation procedure.

Example 3.3

Consider the matrix

$$
M(s)=\left[\begin{array}{cc}
s^{4}+2 s^{3}-5 s-4 & 2 s^{3}+2 s^{2}-2 s-8 \\
-s^{4}+7 s^{3}+7 s^{2}+14 s+6 & -2 s^{4}-5 s^{3}+s^{2}+3 s \\
-2 s^{3}-s^{2}-17 s-9 & 2 s^{4}+3 s^{3}-s^{2}-s-2 \\
2 s^{4}+3 s^{3}-s^{2}-9 s-4 & 0 \\
0 & 2 s^{4}+3 s^{3}-s^{2}-9 s-4
\end{array}\right]
$$

which has rank 2 . We will try to use the polynomial echelon form algorithm with $\nu=2$. Then the coefficient matrix $\widetilde{\mathcal{M}}$ becomes

$\widetilde{\mathcal{M}}=\left[\begin{array}{rrrrrrrrrrrrrr}-4 & -8 & -5 & -2 & 0 & 2 & 2 & 2 & 1 & 0 & 0 & 0 & 0 & 0 \\ 6 & 0 & 14 & 3 & 7 & 1 & 7 & -5 & -1 & -2 & 0 & 0 & 0 & 0 \\ -9 & -2 & -17 & -1 & -1 & -1 & -2 & 3 & 0 & 2 & 0 & 0 & 0 & 0 \\ -4 & 0 & -9 & 0 & -1 & 0 & 3 & 0 & 2 & 0 & 0 & 0 & 0 & 0 \\ 0 & -4 & 0 & -9 & 0 & -1 & 0 & 3 & 0 & 2 & 0 & 0 & 0 & 0 \\ \hline 0 & 0 & -4 & -8 & -5 & -2 & 0 & 2 & 2 & 2 & 1 & 0 & 0 & 0 \\ 0 & 0 & 6 & 0 & 14 & 3 & 7 & 1 & 7 & -5 & -1 & -2 & 0 & 0 \\ 0 & 0 & -9 & -2 & -17 & -1 & -1 & -1 & -2 & 3 & 0 & 2 & 0 & 0 \\ 0 & 0 & -4 & 0 & -9 & 0 & -1 & 0 & 3 & 0 & 2 & 0 & 0 & 0 \\ 0 & 0 & 0 & -4 & 0 & -9 & 0 & -1 & 0 & 3 & 0 & 2 & 0 & 0 \\ \hline 0 & 0 & 0 & 0 & -4 & -8 & -5 & -2 & 0 & 2 & 2 & 2 & 1 & 0 \\ 0 & 0 & 0 & 0 & 6 & 0 & 14 & 3 & 7 & 1 & 7 & -5 & -1 & -2 \\ 0 & 0 & 0 & 0 & -9 & -2 & -17 & -1 & -1 & -1 & -2 & 3 & 0 & 2 \\ 0 & 0 & 0 & 0 & -4 & 0 & -9 & 0 & -1 & 0 & 3 & 0 & 2 & 0 \\ 0 & 0 & 0 & 0 & 0 & -4 & 0 & -9 & 0 & -1 & 0 & 3 & 0 & 2\end{array}\right]$

By searching from the top to the bottom, we find that row 8, 9, 13, 14 and 15 are dependent. Of these, row 8,9 and 15 is the largest set of primary dependent row with least order. The number of rows in this set is 3 which corresponds to the dimension of the null-space which means that we do not have to consider any other dependent rows. These primary dependent rows are defined by

$$
\widetilde{F}=\left[\begin{array}{rrrrr|rrrrr|rrrrr}
0 & 1 & 2 & -3 & -1 & 1 & 1 & 1 & 0 & 0 & 0 & 0 & 0 & 0 & 0 \\
-1 & 0 & 0 & 1 & 2 & -2 & 0 & 0 & 1 & 0 & 0 & 0 & 0 & 0 & 0 \\
-3 & -5 & -6 & 9 & 9 & -10 & -1 & 0 & 0 & -1 & 1 & 1 & 0 & 0 & 1
\end{array}\right]
$$


The corresponding polynomial matrix $F(s)$ in polynomial echelon form is

$$
F(s)=\left[\begin{array}{ccccc}
s & s+1 & s+2 & -3 & -1 \\
-2 s-1 & 0 & 0 & s+1 & 2 \\
s^{2}-10 s-3 & s^{2}-s-5 & -6 & 9 & s^{2}-s+9
\end{array}\right]
$$

which is also a minimal polynomial basis for the left null-space of $M(s)$.

\section{Numerical Considerations}

The two algorithms presented in this section have very different numerical properties. Although the algorithm based on Hermite form is easy to understand, no (to the authors knowledge) numerically stable algorithm exists. Simulations have shown that the algorithm to make the basis row-reduced, proposed in (Callier, 1985) and implemented in (Henrion et al., 1997), is numerically unstable.

On the other hand, the algorithm based on polynomial echelon form is both fast and numerically stable. The critical step in the algorithm is the search for primary dependent rows in the matrix $\widetilde{\mathcal{M}}$. The search for dependent rows can be performed by using a numerically stable projection algorithm described in (Chen, 1984), p. 546. First transform $\widetilde{\mathcal{M}}$ to lower triangular form by multiplication from the right with a matrix $L$. The matrix $L$ is obtained by a series of numerically stable Householder transformations ((Golub and van Loan, 1996), Chapter 5). Now the matrix that defines the dependent rows, is easily obtained by solving for $A$ in the equation

$$
A \widetilde{\mathcal{M}} L=0
$$

Since $\widetilde{\mathcal{M}} L$ is lower triangular, $A$ can be obtained by straightforward, numerically stable substitutions (Chen, 1984). This algorithm is implemented in the Polynomial Toolbox, (Henrion et al., 1997) and from simulations the algorithm was validated to be numerically stable.

\section{Relation to Other Residual Generator Design Methods}

This section discusses the relation between the approach described in this chapter and two other design methods for linear residual generation. Also the relation to the concept of parity functions, although not a design method, is covered. It is interesting to find that the questions of minimality and completeness of solution is not at all obvious for other design methods for residual generation.

\subsection{Parity Equations}

Several interpretations of the terms parity equations (or parity relations) and parity functions exist in the fault diagnosis literature. To clarify the meaning 
here, we use the terms polynomial parity equation and polynomial parity functions, which are the type of parity equations/functions defined in (Chow and Willsky, 1984).

The definition of polynomial parity functions becomes

Definition 2 (Polynomial Parity Function). A polynomial parity function is a function $h(u(t), y(t))$ that can be written as

$$
h(u(t), y(t))=A(s) y(t)+B(s) u(t)
$$

where $A(s)$ and $B(s)$ are polynomial vectors (or matrices if multidimensional parity functions are considered) in $s$. The value of the function is zero if no faults are present.

A polynomial parity equation is then a polynomial parity function set to zero, i.e. $h(u(t), y(t))=0$.

Remark: Parity equations that are not polynomial are often mentioned in the literature: e.g. ARMA parity equations (Gertler, 1991), dynamic parity relations (Gertler and Monajemy, 1995). In accordance with standard mathematical notion, these should be called rational parity equations. A rational parity function is then identical with a linear residual generator.

Parity equations/functions are not a design method; it is solely equations/functions with specific properties. Nevertheless there is a strong relationship between the minimal polynomial approach and polynomial parity functions. For any choice of $\phi(s)$ in (4), $F(s)$ will be a parity function. Thus the minimal polynomial basis approach to residual generator design is then a design method for polynomial parity functions.

Another well known algorithm for finding parity relations is the ChowWillsky scheme (Gertler, 1991). It is shown by example in (Frisk, 1998) that in a numerically demanding design situation, the basic Chow-Willsky approach encounters severe numerical problems whereas the minimal polynomial produces a feasible solution which indicates numerical soundness of the method. Relations to the Chow-Willsky scheme are addressed in more detail in (Nyberg and Frisk, 1999).

\subsection{Frequency Domain Approaches}

A number of design methods described in literature are called Frequency Domain Methods where the residual generators are designed with the help of different transfer matrix factorization techniques. Examples are (Frank and Ding, 1994) for the general case with disturbances and (Ding and Frank, 1990; Viswanadham, Taylor and Luce, 1987) in the non-disturbance case. The methods can be summarized as methods where the residual generator is parameterized as

$$
r=R(s)[\tilde{D}(s)-\tilde{N}(s)]\left(\begin{array}{l}
y \\
u
\end{array}\right)=R(s)(\tilde{D}(s) y-\tilde{N}(s) u)
$$


where $\tilde{D}(s)$ and $\tilde{N}(s)$ form a left co-prime factorization of $G(s)$ over $\mathcal{R H}_{\infty}$, i.e. the space of stable real-rational transfer matrices. Note the close relationship with (12) where the factorization is performed over polynomial matrices instead of over $\mathcal{R H}_{\infty}$.

Inserting (2) into (15) and, as before, assuming $f=0$, gives

$$
r=R(s) \tilde{D}(s) H(s) d
$$

Therefore to achieve disturbance decoupling, the parameterization transfer matrix $R(s)$, must be belong to the left null-space of $\tilde{D}(s) H(s)$, i.e.

$$
R(s) \tilde{D}(s) H(s)=0
$$

Here, note the close connection with $\widetilde{M}_{2}(s)$ in (13). This solution however does not generally generate a minimum order residual generator. In (Ding and Frank, 1990) and (Frank and Ding, 1994), the co-prime factorization is performed via a minimal state-space realization of the complete system, including the disturbances as in (7). This results in $\tilde{D}(s)$ and $\tilde{N}(s)$ of order $n$ that in the general case is larger than the minimal order of decoupling filters. Thus, to find a basis of lowest order that spans all residual generators $Q(s)=R(s)[\tilde{D}(s)-\tilde{N}(s)]$ extra care is required since "excess" states need to be canceled. Note that the polynomial basis approach on the other hand, has no need for cancelations and is in this sense more elegant.

\section{Conclusions}

An algorithm for design of residual generators has been developed with focus on two main issues, not explicitly addressed previously in connection with fault diagnosis, completeness of solution, i.e. a method able to generate all residual generators, and minimality, i.e. a method generating solutions where those of minimal order is trivially extracted from the algorithm output. Other well known design algorithms is explored regarding these properties and it is found that completeness of solution and minimality is not obvious for these design methods.

To be able to deal with these two issues, the residual generation problem was, in Section 2, transformed into finding a minimal polynomial basis for a rational null-space. The completeness of solution was handled in the polynomial framework and proven in Theorem 2. Also, the use of minimal bases assures that minimal-order residual generators are trivially extracted from the output of the algorithm.

An important step in the algorithm is the transformation from a rational problem into a polynomial problem, i.e. to find the left null-space of a polynomial matrix instead of a rational matrix. The transformation was done in two cases: when the system model was given in transfer function form and when the system model was given in state-space form. In the former case, the transformation consists of an MFD and in the latter it was proven in Theorem 3 that a polynomial matrix, the system matrix, can be used for this purpose. 
Further, the importance of a numerically stable design algorithm is handled by utilizing established numerical theory and existing numerical tools.

In conclusion, a residual generator design algorithm has been developed where all residual generators, including those of minimal order, can be systematically constructed with numerically efficient tools.

\section{References}

Callier, F. (1985), 'On polynomial matrix spectral factorization by symmetric factor extraction', IEEE Trans. Automatic Control 30(5), 453-464.

Chen, C.-T. (1984), Linear System Theory and Design, Holt, Rinehart and Winston, New York.

Chow, E. and Willsky, A. (1984), 'Analytical redundancy and the design of robust failure detection systems', IEEE Trans. on Automatic Control 29(7), 603-614.

Ding, X. and Frank, P. (1990), 'Fault detection via factorization approach', Systems $\&$ control letters 14(5), 431-436.

Forney, G. (1975), 'Minimal bases of rational vector spaces, with applications to multivariable linear systems', SIAM J. Control 13(3), 493-520.

Frank, P. and Ding, X. (1994), 'Frequency domain approach to optimally robust residual generation and evaluation for model-based fault diagnosis', Automatica 30(5), 789-804.

Frisk, E. (1998), Residual Generation for Fault Diagnosis: Nominal and Robust Design, Licentiate thesis LIU-TEK-LIC-1998:75, Linköping University.

Gertler, J. (1991), Analytical redundancy methods in fault detection and isolation; survey and synthesis, in 'IFAC Fault Detection, Supervision and Safety for Technical Processes', Baden-Baden, Germany, pp. 9-21.

Gertler, J. and Monajemy, R. (1995), 'Generating directional residuals with dynamic parity relations', Automatica 31(4), 627-635.

Golub, G. and van Loan, C. (1996), Matrix Computations, third edition edn, John Hopkins.

Henrion, D., Kraffer, F., Kwakernaak, H., M.Sebek, S. P. and Strijbos, R. (1997), The Polynomial Toolbox for Matlab, URL: www.math.utwente.nl/polbox/.

Kailath, T. (1980), Linear Systems, Prentice-Hall.

Kung, S., Kailath, T. and Morf, M. (1977), Fast and stable algorithms for minimal design problems, in 'Int. Symp. on Multivariable Technological Systems', IFAC, pp. 97-104. 
Lancaster, P. and Tismenetsky, M. (1985), The theory of matrices, 2nd edn, Academic Press.

Liu, B. and Si, J. (1997), 'Fault isolation filter design for linear time-invariant systems', IEEE Trans. Automatic Control 42(5), 704-707.

Magni, J. and Mouyon, P. (1994), 'On residual generation by observer and parity space approaches', IEEE Trans. on Automatic Control 39(2), 441-447.

Massoumnia, M., Verghese, G. and Willsky, A. (1989), 'Failure detection and identification', IEEE Trans. on Automatic Control AC-34(3), 316-321.

Nikoukhah, R. (1994), 'Innovations generation in the presence of unknown inputs: Application to robust failure detection', Automatica 30(12), 18511867.

Nyberg, M. and Frisk, E. (1999), A minimal polynomial basis solution to residual generation for linear systems, in 'To appear in preprints of IFAC WorldCongress'99', Beijing, P.R. China.

Nyberg, M. and Nielsen, L. (1997), Parity functions as universal residual generators and tool for fault detectability analysis, in 'IEEE Conf. on Decision and Control'.

Patton, R., Frank, P. and Clark, R., eds (1989), Fault diagnosis in Dynamic systems, Systems and Control Engineering, Prentice Hall.

Patton, R. and Kangethe, S. (1989), Robust Fault Diagnosis using Eigenstructure Assignment of Observers, in Patton, Frank and Clark (1989), chapter 4 .

Rosenbrock, H. (1970), State-Space and Multivariable Theory, Wiley, New York.

Viswanadham, N., Taylor, J. and Luce, E. (1987), 'A frequency-domain approach to failure detection and isolation with application to GE-21 turbine engine control systems', Control - Theory and advanced technology $\mathbf{3}(1), 45-72$.

White, J. and Speyer, J. (1987), 'Detection filter design: Spectral theory and algorithms', IEEE Trans. Automatic Control AC-32(7), 593-603.

Wünnenberg, J. (1990), Observer-Based Fault Detection in Dynamic Systems, $\mathrm{PhD}$ thesis, University of Duisburg.

\section{A Useful Properties from Linear Systems The- ory}

This appendix is included to serve as a compilation of definitions, theorems, and basic properties of linear systems, polynomial matrices, and polynomial bases 
used in this thesis. Books describing these matters in detail are e.g. (Kailath, 1980) for a control oriented view, and (Lancaster and Tismenetsky, 1985) for a purely mathematical view.

\section{A.1 Properties of Linear Systems}

Theorem 7 (PBH Rank Test (Kailath, 1980) p. 136). A pair $\{A, B\}$ will be controllable if and only if the matrix

$$
\left[\begin{array}{ll}
s I-A & B
\end{array}\right] \text { has rank } n \text { for all } s
$$

\section{A.2 Properties of Polynomial Matrices}

To avoid unnecessary misunderstandings: a polynomial matrix, which in some literature is called matrix polynomials (Lancaster and Tismenetsky, 1985), is any matrix $F(s)$ where the individual elements are scalar polynomials in $s$. Here, the co-efficients in the polynomials will always be real.

Definition 3 (Normal Rank). The (normal) rank of a polynomial matrix $F(s)$ is the largest rank $F(s)$ has for any $s \in \mathbb{C}$.

Sometimes the word normal is omitted, when the text only says rank it is always meant normal rank.

Definition 4 (Row-reduced Matrix). Consider a polynomial matrix $F(s)$ with row-degrees $\mu_{i}$. It is always possible to write

$$
F(s)=S(s) D_{h c}+L(s)
$$

where

$$
\begin{aligned}
S(s) & =\operatorname{diag}\left\{s^{\mu_{i}}, i=1, \ldots, m\right\} \\
D_{h c} & =\text { the highest-row-degree coefficient matrix } \\
L(s) & =\text { the remaining term with row-degrees strictly less than those of } F(s)
\end{aligned}
$$

A full row rank matrix $F(s)$ is said to be row-reduced if its leading row-coefficient matrix has full row rank.

Definition 5 (Irreducible and Unimodular Matrices). A polynomial matrix $F(s)$ is said to be irreducible if it has full rank for all finite s. If $F(s)$ is irreducible and square it is said to be unimodular. A unimodular matrix has a unimodular inverse.

Theorem 8 (Polynomial Solutions to Polynomial Equations (Kailath, 1980)). Let $F(s)$ be a full row rank polynomial matrix, and let $p(s)$ be a given polynomial vector. Then the equation

$$
q(s) F(s)=p(s)
$$

will have a polynomial solution if and only if $F(s)$ is irreducible. 


\section{A.3 Properties of Polynomial Bases}

Definition 6 (Degree of a Polynomial Vector). The degree of a polynomial vector is the highest degree of all the entries of the vector. If the vector is a rowvector, it is called row-degree.

Definition 7 (Order of a Polynomial Basis). Consider a polynomial basis $F(s)$ with row-degrees $\mu_{i}$. Then the order of the basis is defined as

$$
\operatorname{order}(F(s))=\sum_{i} \mu_{i}
$$

Theorem 9 (Minimal Bases). Consider a full row (normal) rank polynomial matrix $F(s)$. Then the following statements are equivalent

- The rows of $F(s)$ form a minimal basis for the rational vector space they generate.

- $F(s)$ is row-reduced and irreducible.

- $F(s)$ has minimal order.

Theorem 10 (Irreducible Basis). If the rows of $F(s)$ is an irreducible polynomial basis for a space $\mathcal{F}$, then all polynomial row vectors $f(s) \in \mathcal{F}$ can be written $f(s)=\phi(s) F(s)$ where $\phi(s)$ is a polynomial row vector.

Proof. Since $F(s)$ is a basis, all $f(s) \in \mathcal{F}$ can be written $f(s) g(s)=\phi(s) F(s)$. For each root $\alpha$ of $g(s)$ it holds that

$$
f(\alpha) g(\alpha)=\phi(\alpha) F(\alpha)=0
$$

Since $F(s)$ is irreducible, it has full row rank for all $s$ and in particular $s=\alpha$. This implies that $\phi(\alpha)=0$, i.e. all roots of $g(s)$ are also roots of $\phi(s)$. Thus $\phi(s)$ can be factorized as $\phi(s)=g(s) \bar{\phi}(s)$ and

$$
f(s) g(s)=g(s) \bar{\phi}(s) F(s)
$$

This implies

$$
f(s)=\bar{\phi}(s) F(s)
$$

